\title{
Schweres refraktäres eosinophiles Asthma
}

_ Patienten mit schwerem refraktärem eosinophilem Asthma erleben häufiger Exazerbationen, die eine stationäre Behandlung erforderlich machen. Seit Februar 2016 steht für diese Patienten der erste zugelassene monoklonale Antikörper gegen Interleukin-5 (IL-5) als Zusatztherapie zu einer bestehenden Asthmamedikation zur Verfügung.

Um den richtigen Patienten für die neue Therapieoption Mepolizumab $\left(\right.$ Nucala $\left.^{\circledR}\right) \mathrm{zu}$ identifizieren, müsse zunächst eine korrekte Differenzierung zwischen schwerem und schwierig zu behandelndem Asthma getroffen werden, erläuterte Dr. Cordula Mohrlang, Pneumologin bei GSK Deutschland. Die European Respiratory Society und die American Thoracic Society sprechen von schwerem Asthma, wenn die Beschwerden nur mit hohen Dosen inhalativer Kortikosteroide und weiteren Kontroll-Medikamenten und/oder systemischen Kortikoiden kontrolliert werden können oder trotz dieser Therapie unkontrolliert bleiben [Chung KF et al. 2014].

Schätzungsweise $60 \%$ der Patienten mit schwerem Asthma haben eine eosinophile Entzündung der Atemwege. Ihnen könnte der monoklonale IL-5-Antikörper helfen. Er senkt die Rate der Exazerbationen, verbessert die Lebensqualität und reduziert den Bedarf an oralen Kortikoiden, wie ein Phase-IIb/ III-Studienprogramm belegt hat $(\mathrm{Pa}-$ vord ID et al. Lancet. 2012; Ortega HG et al. NEJM. 2014; Bel EH et al. NEJM. 2014].

Der Einsatz von Mepolizumab sollte in Erwägung gezogen werden, wenn ein schweres refraktäres Asthma bestätigt ist, eine Eosinophilie zur Sicherung des

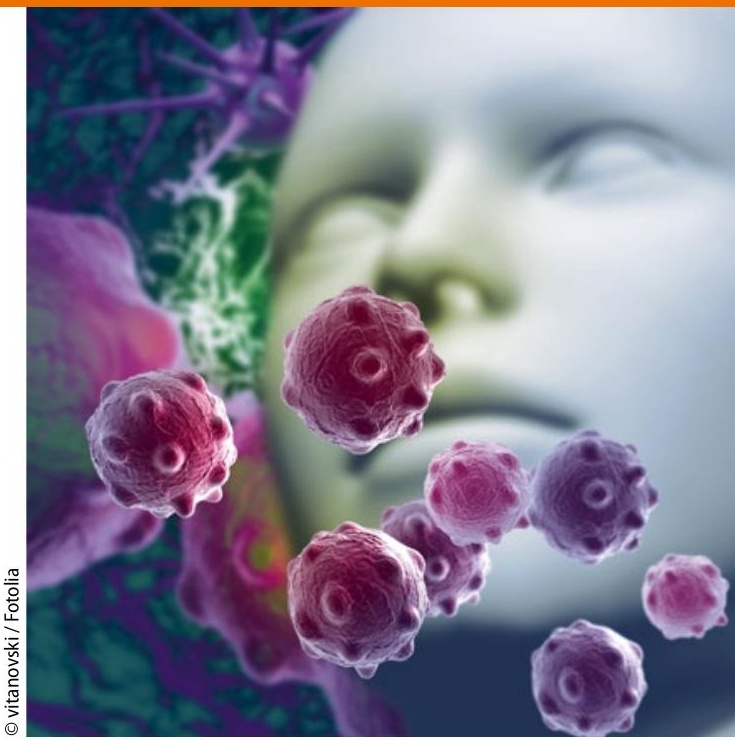

Allergene lassen sich nicht beseitigen allergische Beschwerden schon.

eosinophilen Phänotyps nachgewiesen wurde und ein allergisches Asthma bronchiale oder ein anderer Phäntotyp ausgeschlossen wurde.

Dagmar Jäger-Becker

- Launch-Pressekonferenz „Nucala ${ }^{\oplus}$ - Die erste gezielte AntiIL-5-Therapie für Patienten mit schwerem refraktärem eosinophilem Asthma"; München, Dezember 2015 (Veranstalter: GlaxoSmithKline)

\section{Versagen der basal unterstützten oralen Insulintherapie}

\section{Degludec/Liraglutid-Fixkombination als Therapiealternative}

— „Deutschland hat in Europa den höchsten Pro-Kopf-Insulinverbrauch, ohne eine bessere Einstellung der Diabetiker zu erreichen", erklärte PD Dr. Elmar Jaeckel, Hannover. Eine intensivierte Insulintherapie bedeute aber fast zwangsläufig Gewichtszunahme und eine Steigerung der Hypoglykämiegefahr. Hinzu kommen häufiges Spritzen, häufiges Messen des Blutzuckers und eine schlechte Planbarkeit des Alltags.

Menschen mit Diabetes fühlen sich hierdurch in ihrer Leistungsfähigkeit und Stimmung beeinträchtigt, sie empfinden Stress - so das Ergebnis einer von Novo Nordisk initiierten Online-Patientenbefragung. Viele Betroffene zögern deswegen notwendige Therapieintensivierungen lange hinaus. Dabei gibt es heute Alternativen, z. B. die Kombination aus Basalinsulin plus GLP-1-Analogon. Seit Mitte 2015 steht die Fixkombination Xultophy ${ }^{\circledR}$ zur Verfügung, bestehend aus Insulin degludec und Liraglutid, kurz IDegLira.

\section{Deutliche Vorteile}

Die Fixkombination führt gegenüber intensivierten Insulintherapien zu einer vergleichbaren Blutzuckersenkung, niedrigerem Blutdruck, weniger Gewicht und einer Senkung der Hypoglykämierate.

Zudem ist die Therapie viel einfacher: Der Patient mit intensivierter Insulintherapie spritzt 4-mal täglich, pro Woche misst er 28-mal seinen Blutzucker. Die basal unterstützte Inkretin-Therapie erfordert nur eine Injektion täglich, zu einem beliebigen Zeitpunkt unabhängig von der Mahlzeit, sowie drei Blutzuckerkontrollen pro Woche.

Jaeckel empfiehlt eine langsame Auftitrierung. Dies bestätigen die Zulassungsstudien DUAL II [Gough S et al.; Lancet Diabetes Endocrinol. 2014;2:88593] und DUAL V [Buse et al. ADA 2015]. Jaeckel: „Meine Patienten sind sehr zufrieden mit dieser Therapie. Sie lässt ihnen Freiheiten, die Titration ist denkbar einfach, die Wirkung ist stark und die Patienten nehmen ab. Ich halte das für eine wichtige neue Option in der Diabetestherapie."

\section{Dr. Dirk Einecke}

- Pressekonferenz "Studienergebnisse und Praxiserfahrungen: Medizinischer Fortschritt am Beispiel von IDegLira"; Frankfurt, Januar 2016 (Veranstalter: Novo Nordisk) 